\title{
SARS-CoV-2 infection and the COVID-19 pandemic: a call to action for therapy and interventions to resolve the crisis of hospitalization, death, and handle the aftermath
}

Peter A. McCullough ${ }^{1,2,3, *}$, Kris Vijay ${ }^{4}$

${ }^{1}$ Baylor University Medical Center, Dallas, TX 75226, USA

${ }^{2}$ Baylor Heart and Vascular Institute, Dallas, TX 75226, USA

${ }^{3}$ Baylor Jack and Jane Hamilton Heart and Vascular Hospital, Dallas, TX 75226, USA

${ }^{4}$ Institute of Congestive Heart Failure at Abrazo Arizona Heart Hospital and Heart Institute, Phoenix, AZ 85016, USA

*Correspondence: peteramccullough@gmail.com (Peter A. McCullough)

DOI:10.31083/j.rcm.2021.01.301

This is an open access article under the CC BY 4.0 license (https://creativecommons.org/licenses/by/4.0/).

Submitted: 23 February 2021 Accepted: 24 February 2021 Published: 30 March 2021

The SARS-CoV-2 (COVID-19) pandemic has been the greatest challenge to medical practice in our lifetimes. Among vast populations, there has been fear, panic, confusion, division, and a wide array of regulatory and public health responses to the crisis [1]. We believe it is important for patients and physicians to keep in mind this pandemic is an emergency crisis and is not a usual context for public health measures, drug development, guidelines, and recommendations for patient practice [2].

In this bold issue of Reviews in Cardiovascular Medicine, we present the most comprehensive and scalable early ambulatory treatment program for high-risk patients who have contracted SARS-CoV-2 [3]. No such published regimen has received so much acclaim and the concepts embodied in this paper have been responsible for saving hundreds of thousands of lives and sparing millions of hospitalization $[4,5]$. Since it took months for the large body of information to congeal on the pathophysiology and rationale for treatment, we cannot go back in time and estimate avoidable losses [6]. Going forward, available sources of data from Zelenko and Procter, suggest available drugs used in combination can reduce hospitalizations and death by $85 \%$ and that no high-risk patient with COVID-19 should go untreated at home [7, 8]. Lack of home treatment can only lead to long durations of illness, more intense symptoms, and increases the risk of hospitalization, death, and potentially post-COVID-19 syndromes. At this point in time, there is no justification in any part of the world for denial of available, appropriately prescribed off label intracellular anti-infectives, corticosteroids/colchicine, and antithrombotics.

In this issue we address the interface between important micronutrient deficiencies including vitamin $\mathrm{D}$, endothelial dysfunction, and poor outcomes $[9,10]$. These and other papers are the basis for the "nutraceutical bundle" recommended by our comprehensive treatment guidance. The cardiovas- cular system is affected in COVID-19 and we explore the manifestations and potential therapeutics for SARS-CoV-2 myocardial injury [11]. Additionally we highlight the postCOVID-19 issues of protracted symptoms and need for rehabilitation [12]. It is our hope with more early ambulatory treatment and the avoidance of prolonged and severe symptoms, that rates and intensity of post-COVID-19 syndromes declines over time.

Finally we put forward some important societal and public health issues for the readers to consider. We are deeply concerned that the peer-review process has become politicized with major journals making overt statements about political candidates and original papers clearly slanting interpretation of results to further political gain [13]. This hurts science, physicians, and most importantly patients [14]. We also pick up on the value of public health interventions and technologies which have never been attempted in the modern era including telemedicine, machine learning, travel restrictions, isolation, and lockdowns [15-18].

In summary, Fig. 1 demonstrates that the largest public health gain in terms of hospitalization and death benefit is for early multidrug regimens for high risk patients who are acutely ill with COVID-19. This population should be our highest priority and should be tended to with patient treatment guides, immediate access to research protocols, and engagement with physicians either by telemedicine or inperson who are familiar with the signals of benefit and the safety information available for these commonly prescribed drugs [19]. Access to monoclonal antibodies available under Emergency Use Authorization should be ensured and featured by emergency departments, urgent care clinics, and nursing homes at the point of care where high-risk patients receive a positive SARS-CoV-2 result [3]. It is our sincere hope that this issue of Reviews in Cardiovascular Medicine will touch and save more lives than any of our prior efforts. The 
SARS-CoV-2 pandemic and the COVID-19 crisis has been a call to arms for all medical professionals and it is our highest calling to respond to acutely ill patients in their greatest time of need.

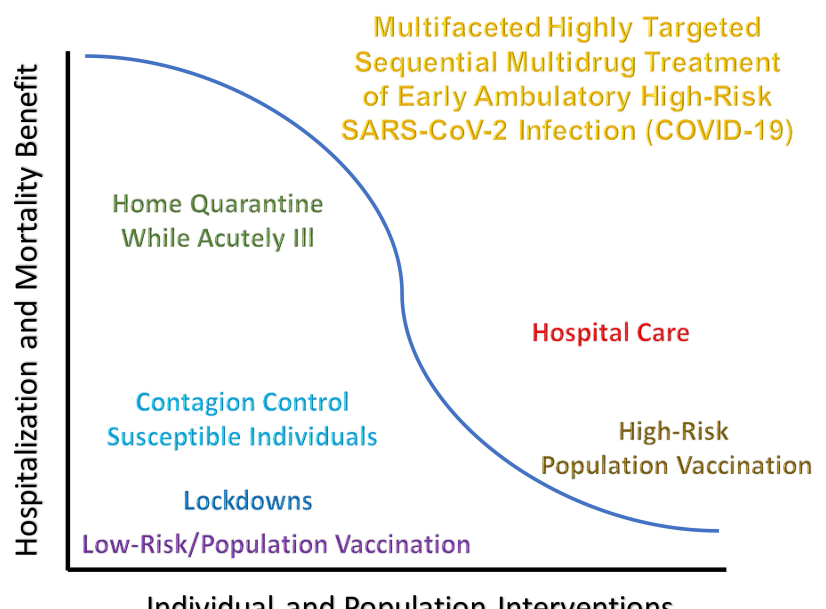

Fig. 1. Relative benefit of reduction in COVID-19 hospitalization for individual and population interventions taken as part of the pandemic response.

\section{Author contributions}

Both authors contributed to the drafting and finalization of the manuscript.

\section{Ethics approval and consent to participate}

Ethics approval is not applicable.

\section{Acknowledgment}

There are no acknowledgments to disclose.

\section{Funding}

This research received no external funding.

\section{Conflict of interest}

The authors declare no conflict of interest.

\section{References}

[1] McCullough PA, Eidt J, Rangaswami J, Lerma E, Tumlin J, Wheelan $\mathrm{K}$, et al. Urgent need for individual mobile phone and institutional reporting of at home, hospitalized, and intensive care unit cases of SARS-CoV-2 (COVID-19) infection. Reviews in Cardiovascular Medicine. 2020; 21: 1-7.

[2] McCullough PA, Oskoui R. Early multidrug regimens in new potentially fatal medical problems. Reviews in Cardiovascular Medicine. 2020; 21: 507-508.
[3] McCullough PA, Alexander PE, Armstrong R, Arvinte C, Bain AF, Bartlett RP, et al. Multifaceted highly targeted sequential multidrug treatment of early ambulatory high-risk SARS-CoV-2 infection (COVID-19). Reviews in Cardiovascular Medicine. 2020; 21: 517530.

[4] McCullough PA, Kelly RJ, Ruocco G, Lerma E, Tumlin J, Wheelan $\mathrm{KR}$, et al. Pathophysiological basis and rationale for early outpatient treatment of SARS-CoV-2 (COVID-19) infection. American Journal of Medicine. 2021; 134: 16-22.

[5] McCullough PA. Favipiravir and the need for early ambulatory treatment of SARS-CoV-2 infection (COVID-19). Antimicrobial Agents and Chemotherapy. 2020; 64: e02017-e02020.

[6] Procter BC, Ross C, Pickard V, Smith E, Hanson C, McCullough PA. Clinical outcomes after early ambulatory multidrug therapy for high-risk SARS-CoV-2 (COVID-19) infection. Reviews in Cardiovascular Medicine. 2020; 21: 611-614.

[7] Derwand R, Scholz M, Zelenko V. COVID-19 outpatients: early risk-stratified treatment with zinc plus low-dose hydroxychloroquine and azithromycin: a retrospective case series study. International Journal of Antimicrobial Agents. 2020; 56: 106214.

[8] Procter C, Ross C, Pickard V, Smith E, Hanson C, McCullough PA, et al. Early ambulatory multidrug therapy reduces hospitalization and death in high-risk patients with SARS-CoV-2 (COVID-19). Authorea. (in press)

[9] Zhang J, McCullough PA, Tecson KM. Vitamin D deficiency in association with endothelial dysfunction: implications for patients with COVID-19. Reviews in Cardiovascular Medicine. 2020; 21 339-344.

10] Zhang J, Tecson KM, McCullough PA. Endothelial dysfunction contributes to COVID-19-associated vascular inflammation and coagulopathy. Reviews in Cardiovascular Medicine. 2020; 21: 315-319.

[11] Mahenthiran AK, Mahenthiran AK, Mahenthiran J. Cardiovascular system and COVID-19: manifestations and therapeutics. Reviews in Cardiovascular Medicine. 2020; 21: 399-409.

[12] Thamman R, Janardhanan R. Cardiac rehabilitation using telemedicine: the need for tele cardiac rehabilitation. Reviews in Cardiovascular Medicine. 2020; 21: 497-500.

[13] Venegas-Vera AV, Colbert GB, Lerma EV. Positive and negative impact of social media in the COVID-19 era. Reviews in Cardiovascular Medicine. 2020; 21: 561-564.

[14] McCullough PA. Regarding: "Hydroxychloroquine: a comprehensive review and its controversial role in coronavirus disease 2019”. Annals of Medicine. 2021; 53: 286-286.

[15] Zimmerman A, Kalra D. Usefulness of machine learning in COVID-19 for the detection and prognosis of cardiovascular complications. Reviews in Cardiovascular Medicine. 2020; 21: 345352.

16] Colbert GB, Venegas-Vera AV, Lerma EV. Utility of telemedicine in the COVID-19 era. Reviews in Cardiovascular Medicine. 2020; 21: 583-587.

[17] Suri JS, Puvvula A, Majhail M, Biswas M, Jamthikar AD, Saba L, et al. Integration of cardiovascular risk assessment with COVID-19 using artificial intelligence. Reviews in Cardiovascular Medicine. 2020; 21: 541-560.

[18] Burlacu A, Crisan-Dabija R, Covic A, Raiu C, Mavrichi I, Popa IV, et al. Pandemic lockdown, healthcare policies and human rights: integrating opposed views on COVID-19 public health mitigation measures. Reviews in Cardiovascular Medicine. 2020; 21: 509516.

[19] Association of American Physicians and Surgeons. Association of American Physicians and Surgeons. 2021. Avaliable at: https://aa psonline.org/ (Accessed: 23 February 2021). 